\title{
Age-related injury risk curves for the lumbar spine for use in low-back-pain prevention in manual handling tasks
}

\author{
Naoki Kudo, Yoji Yamada* and Daisuke Ito
}

\begin{abstract}
This study introduces injury risk curves for the lumbar spine for use in the risk assessment of low back pain (LBP) caused by manual lifting of heavy loads. LBP has been a longstanding problem among industrial workers, giving rise to the development of assistive devices. However, quantitative evaluation methods to verify the safety of such devices have not yet been established. The notable biomechanical criterion of $3.4 \mathrm{kN}$ of lumbar compressive force, defined by the National Institute for Occupational Safety and Health, applies only to young, healthy workers with a fixed risk level. This study on injury risk curves clarified the risk level of injury to the lumbar spine due to lumbar compressive force for individuals within a wide age range. The findings can be applied for the design and evaluation of assistive devices as well as the design of ergonomic guidelines for manual work.
\end{abstract}

Keywords: Low back pain, Risk curve, Manual lifting task, Compressive strength

\section{Introduction}

Low back pain (LBP) is one of the major occupationrelated diseases. It is considered that the manual lifting of heavy loads can increase the risk of LBP in various occupational fields. Caregiving is one of the most frequent fields where work-related LBP occurs [1]. Caregivers often transfer elderly persons from one place to another, which is classified as a manual lifting task. As one of the solutions, certain physical assistive devices have been developed to prevent these workers from developing LBP $[2,3]$. However, the standardization of methods for the quantitative assessment of the safety and effectiveness of these devices against LBP has just been established [4]. Current research aims to contribute to the establishment of quantitative evaluation methods for verifying the safety of such devices.

To prevent LBP among industrial workers, the National Institute for Occupational Safety and Health (NIOSH) has provided guidelines for manual lifting tasks. These

\footnotetext{
*Correspondence: yoji.yamada@mae.nagoya-u.ac.jp Department of Mechanical Systems Engineering, Graduate School of Engineering, Nagoya University, Furo-Cho, Chikusa-ku, Nagoya 464-8603, Japan
}

guidelines currently include the NIOSH lifting equation (NLE), which provides estimates of the recommended weight limit (RWL) in hands [5, 6]. The 1991 revised version of the NLE (RNLE) has brought widespread impact that it is adopted as an ergonomic standard for use by various local, state, and international entities [7]. In the guideline, a limit of $3.4 \mathrm{kN}$ compressive force on the lumbar spine was introduced as a biomechanical criterion to assess the risk of LBP. This criterion was first proposed in the 1981 original NLE and was maintained in the RNLE $[5,6]$. Until now, it is widely used as a major criterion for the assessment of manual lifting tasks [8-15].

The NIOSH biomechanical criterion is based on studies on the compressive strength (CS) of lumbar spine specimens from human cadavers $[5,6]$. It is claimed that CS, which has presented the strongest relationship with chronic LBP in controlled scientific studies, is a valid index for the prevention of disc degeneration [16]. When a disc degenerates, it causes inflammation, which irritates the pain receptors in the outer areas of the disc [17]. Disc degeneration has been also associated with the occurrence of herniated discs [18]. Moreover, endplate fracture is considered to initiate disc degeneration [19] (Fig. 1). The endplate breaks when the spine experiences 


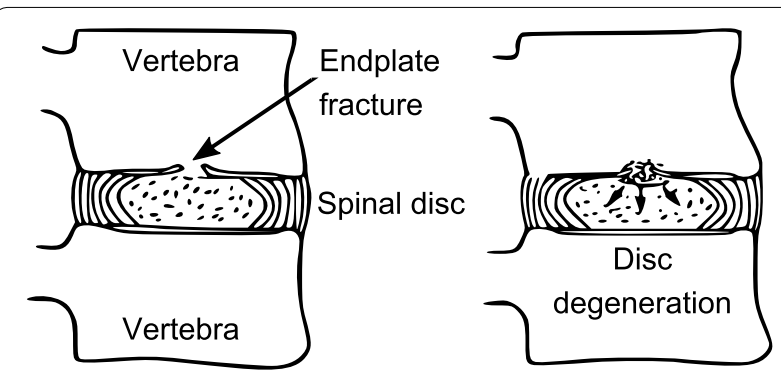

Fig. 1 End-plate fracture initiates disc degeneration (adapted from the work of Bogduk [17])

a sufficient load, as the endplate is the weakest part in the spine, and it is the first structure to fail under a heavy load [18]. Therefore, the value of CS determines the compressive force limit on the lumbar spine.

As no exact value for CS can be found in the NIOSH literatures, the risk level for the biomechanical criterion of $3.4 \mathrm{kN}$ seems to be valid for approximately $20-30 \%$ of the general population [6]. According to the Work Practices Guide for Manual Lifting [5], this criterion applies to young, healthy workers. Meanwhile, using linear regression analysis, Genaidy et al. [20] showed that older individuals have significantly lower CS. Furthermore, Jäger and Luttmann [21] reported that the $3.4 \mathrm{kN}$ criterion cannot be applied for a wide range of individuals, and suggested that the criterion be limited to individuals up to 40 years of age. Arjmand et al. [12] have also recommended to revisit the criterion for these reasons.

The present study aimed to quantitatively assess the risk level of compressive force on the lumbar spine to verify safety in using assistive devices and in performing manual handling tasks without using the devices. Although Genaidy et al. [20] performed risk analysis using CS values from several studies, their proposed equations were linear. However, the risks are known to be expressed as nonlinear risk curves, characterized by nonlinearity for lower or higher risk levels and which can be overestimated or underestimated with the linear form $[22,23]$. Thus, the use of assistive devices may help control the amount of compressive force on the lumbar spine during manual lifting tasks. For the evaluation of such devices, the accuracy of estimation in the lower risk is important. Therefore, the analysis of a low risk level, which cannot be expressed in a linear form, should be performed. This study introduces nonlinear injury risk curves derived from nonparametric statistical analyses, which are applicable to individuals within a wide age range.

\section{Factors affecting CS}

Consideration of individual differences in parameter characteristics in the risk analysis reduces variance of data and can improve the precision of estimating the risks. In this section, we choose one parameter based on linear regression analysis.

\section{Mechanical aspects of CS}

Some studies suggest that bone mineral density (BMD), bone mineral content (BMC), and endplate area are mechanical factors affecting the CS value. Biggemann and Hilweg [24] reported a strong correlation of $r=0.84$ between CS and the product of BMD and endplate area. In another study by Hansson et al. [25], the correlation between CS and BMC was $r=0.86$. The units of measurement for BMD and BMC are $\mathrm{g} / \mathrm{cm}^{2}$ and $\mathrm{g}$, respectively, and their respective values can be interpreted in the same manner. Using BMD multiplied by endplate area and $\mathrm{BMC}$ as parameters for risk estimation may be more appropriate in terms of accuracy; however, these methods are difficult to employ. The major drawback is that these methods require X-ray measurements, which involve radiation risk.

\section{Parameters considering ease of implementation}

The correlation between CS and other parameters that are relatively easy to measure is required for precision purposes. Genaidy et al. [20] performed multiple regression analysis and proposed a linear equation for estimating the CS value using age, gender, lumbar motion segment (levels of lumbar segment), and body weight as parameters. They also reported the following equation, which includes the risk term "population percentile" (PP).

$$
\begin{aligned}
\mathrm{CS}= & 7222.41-(1047.1 \times \mathrm{AGE}) \\
& -(1279.18 \times \text { GENDER })+(56.73 \times \mathrm{PP})
\end{aligned}
$$

CS: compressive strength [N]; AGE: age group (20-29 years $=1,30-39$ years $=2,40-49$ years $=3,50$ years and above $=4)$; GENDER: gender $($ male $=1$, female $=2)$; PP: population percentile $(1,5,10,25,50,74,90,95$ and 99$)$.

Analysis of Eq. 1 does not match our purpose because it is fitted to a linear equation. Our focus is on low risk level. Regarding risk analysis, nonlinear risk curves are used in the field of motor-vehicle crash testing [22, 23].

Increasing the number of parameters restrains the amount of data we can use from the existing literature, which can affect the accuracy of risk estimation. This study focused on clarifying which parameter mostly affects CS; the parameter that is related to BMD, BMC, or endplate area may be more reasonable. As candidates 
of this parameter, age, gender, lumbar motion segment (levels of lumbar segment), and body weight were considered, which were used for the linear equation for the estimation of the CS value that Genaidy et al. [20] proposed. Hansson et al. [25] reported that no relationship was found between BMC and levels of lumbar segment through a regression analysis. By reanalyzing the data that Genaidy et al. [20] used (Hutton and Adams [26]; Hansson et al. [25]; Hansson and Roos [27]; and Adams and Hutton [28]), we computed again the coefficient of determination $R^{2}$ and the results against CS were 0.32548 for age, 0.17225 for gender, and 0.12136 for body weight. The relationship between the BMD or the BMC and age were reported by Ebbesen et al. [29], where $R^{2}$ was 0.43 for the BMD, 0.29 for the BMC, respectively. Considering the above, the age was chosen as the parameter in this work.

\section{Studies on the relationship between CS and age}

By focusing on age as the parameter, we found sources of dataset other than the previously mentioned work used by Genaidy et al. [20] in deriving Eq. 1. Their dataset was also used in the $R^{2}$ analysis in this study. The relationship between CS and age is illustrated in Fig. 2, based on experimental data obtained by Eie [30], Hansson et al. [25], Hansson and Roos [27], Hutton and Adams [26], Adams and Hutton [28], Brinckmann et al. [24, 31], and Ebbesen et al. [29]. The data collected and presented in the figure were used to conduct the risk analysis described in the following section, where the

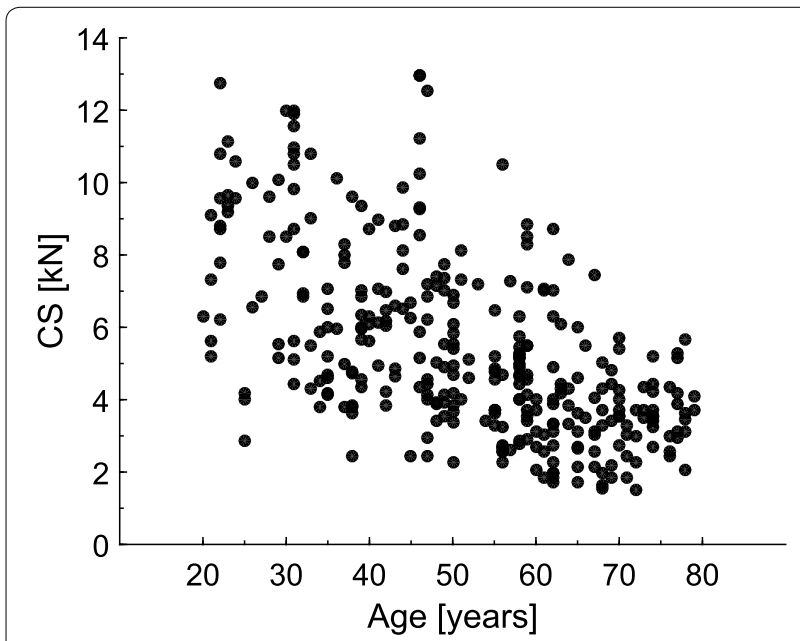

Fig. 2 Compressive strength (CS) of lumbar spine versus age. Data were taken from the works of Eie [30], Hansson et al. [25], Hansson and Roos [27], Hutton and Adams [26], Adams and Hutton [28], Brinckmann et al. [24, 31] and Ebbesen et al. [29]. Data obtained from Ebbesen et al. were read from the scatter plots. The age range was restricted to $20-79$, considering the amount of data and the number of references. $(N=320)$ age values were restricted within 20-79 years due to limited resources on data and references. Moreover, the resources included data from different sections of lumbar segments extracted from the same cadavers, to collect sufficient amount of data for analysis.

It should be noted that experimental conditions for measuring CS were not entirely similar among the above referenced works. One of the differences pertained to the compression of either a single lumbar vertebra or a lumbar motion segment. The lumbar spine consists of five vertebrae (L1, L2, L3, L4, and L5). A lumbar motion segment comprises two lumbar vertebrae and the intervertebral soft tissue, which means that it consists of two lumbar vertebral units [28]. Meanwhile, a vertebra is the single bone component/unit [25]. Hutton and Adams [26], Adams and Hutton [28] and Brinckmann et al. [24, 31] performed analyses for lumbar motion segments, whereas Hansson et al. [25], Hansson and Roos [27], and Ebbesen et al. [29] focused on a group of single lumbar vertebrae. Eie [30] included both lumbar motion segments and single lumbar vertebrae in his experiments. There are certain differences among the experiments on lumbar motion segments in terms of compressive conditions. Hutton and Adams [26] and Adams and Hutton [28] applied loads that bent the joints to the limit of normal flexion, whereas in other studies either the joints did not bend or the researchers did not mention anything about bending of joints in their works. Other differences include the level of compression, and whether a preloading was applied before any increase in compressive force was applied to the limit of fracture. Hansson et al. [25], Hansson and Roos [27], and Ebbesen et al. [29] performed loading in $5 \mathrm{~mm} / \mathrm{min}$, whereas Hutton and Adams [26] performed in $3000 \mathrm{~N} / \mathrm{s}$. Brinckmann et al. [24] applied a preload of $1 \mathrm{kN}$ for $15 \mathrm{~min}$. After the preload was removed, a main loading of $1 \mathrm{kN} / \mathrm{s}$ was applied. Conditions of preserving the specimens were also different. The preserved specimens used by Eie [30] and Brinckmann et al. [24], and some of the preserved specimens used by Hutton and Adams [26] were fresh (never frozen before the tests), whereas others preserved the specimens by initially freezing them (stored in approximately $-20^{\circ} \mathrm{C}$ ) and then thawing them before the tests.

These differences may affect the experimental results. However, it is difficult for us at this stage to consider what to exclude in the data because the condition of applying the compressive force is different enough from that of the other remaining part of the data, and we should continue collecting a larger amount of data under the same conditions for analysis. Even with considering these differences in conditions, it can be observed in Fig. 2 that the CS values tend to decline as the age values increase. 


\section{Risk estimation}

In this section, we will derive the risk curves using data shown in Fig. 2.

\section{Risk curve for the general population}

The kernel density estimation [32] can be used to estimate the smooth probability distribution function $\hat{f}_{h}(x)$ :

$$
\hat{f}_{h}(x)=\frac{1}{n h} \sum_{i=1}^{n} K\left(\frac{x-X_{i}}{h}\right)
$$

Here, $x$ denotes the point of CS at which the density $\hat{f}_{h}(x)$ is estimated, $X_{i}$ denotes the observed CS of sample $i, n$ is the number of samples, $h$ is the bandwidth, and $K(x)$ is the kernel function. In the present study, the normal distribution was used for the kernel:

$$
K(x)=\frac{1}{\sqrt{2 \pi}} e^{-\frac{1}{2} x^{2}} .
$$

Moreover, for the boundary condition that CS should be a positive value, the transformation $x \rightarrow t(x)$ was employed [33], where $t(x)=\ln (x)$ in this study:

$$
\hat{f}_{h}(x)=\frac{1}{n h} \sum_{i=1}^{n} K\left(\frac{t(x)-t\left(X_{i}\right)}{h}\right) t^{\prime}(x) .
$$

Then, the cumulative distribution function, namely the risk curve, is expressed as follows:

$$
\hat{r}_{h}(x)=\int_{-\infty}^{x} \hat{f}_{h}(x) d x .
$$

Equation 6 was used for calculating the bandwidth $h$ given in the work of Bowman and Azzalini [33]:

$$
\begin{aligned}
& h=\left(\frac{4}{3 n}\right)^{1 / 5} \sigma, \\
& \tilde{\sigma}=\operatorname{median}\left\{\left|X_{i}-\tilde{\mu}\right|\right\} / 0.6475,
\end{aligned}
$$

where $\tilde{\sigma}$ is the median of sample $X_{i}$. As the risk curve derived from the kernel method (Eq. 5) requires the input of all the 320 datasets presented in Fig. 2, it is inefficient to use it in this form from the viewpoint of usability. Therefore, we considered fitting it to a function (Eq. 8) that requires inputting only three coefficients:

$$
r(x)=\frac{1}{1+\exp \left(\frac{a_{0}+a_{1} x^{2}+a_{2} x^{3}}{x^{2}}\right)} .
$$

The way how to choose the coefficients in the equation for fitting the risk curve is described in Appendix A. For the fitting operation, the fminunc function in the

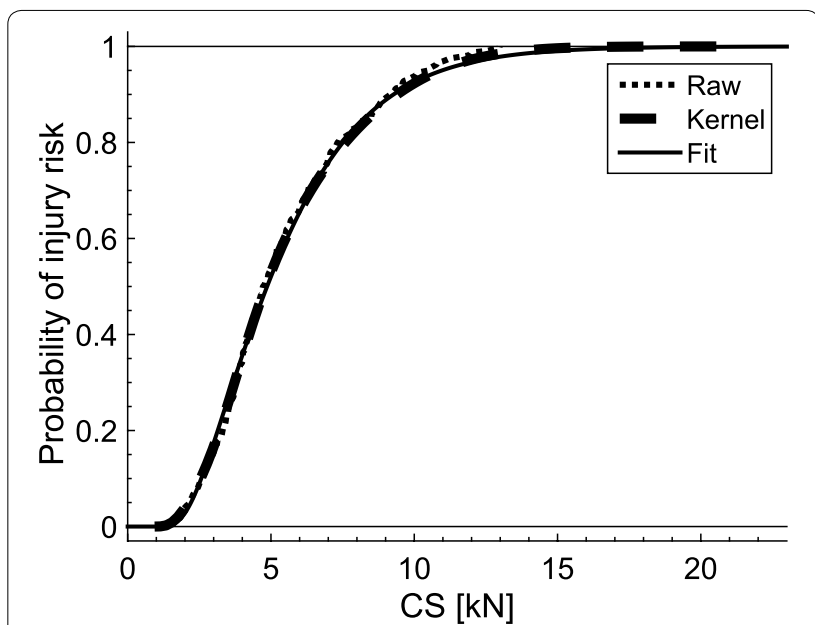

Fig. 3 Risk curves for the age group 20-79 years. CS, compressive strength

Table 1 Resultant coefficients of the risk curve for the general population fitted to Eq. 8

\begin{tabular}{lll}
\hline $\boldsymbol{a}_{\mathbf{0}}$ & $\boldsymbol{a}_{\mathbf{1}}$ & $\boldsymbol{a}_{\mathbf{2}}$ \\
\hline 11.24 & 1.568 & -0.4199 \\
\hline
\end{tabular}

optimization toolbox of MATLAB (MathWorks) was used with the cost function of the least-squares error.

Figure 3 shows the resultant risk curve, whereas Table 1 lists the resultant coefficients fitted to the curves of Eq. 8.

\section{Risk curves for three generations}

To estimate the risk for different generations, the data presented in Fig. 2 were grouped into several generations. By judging through the number of samples for each group, three generation groups (20-year width) were chosen in this paper: $20-39,40-59$, and $60-79$ years. The number of samples was 85, 129 and 106, respectively. For each age group, the risk curves were estimated using the same method described in "Risk curve for the general population" section.

Figure 4 illustrates the risk curves for each age group. Table 2 lists the resultant coefficients fitted to the curves in Eq. 8. The forces estimated via the risk curves associated with a $10 \%$ risk of injury for the age groups $20-39$, $40-69$ and $70-89$ years were $3.72,2.98$, and $2.03 \mathrm{kN}$, respectively (Fig. 5).

\section{Risk curves for specific ages}

In certain cases, the risk curve for a specific age can be more useful than the risk curve for the generations. As an example, the developers of physical assistive devices would be able to set the assist power/rate to change 


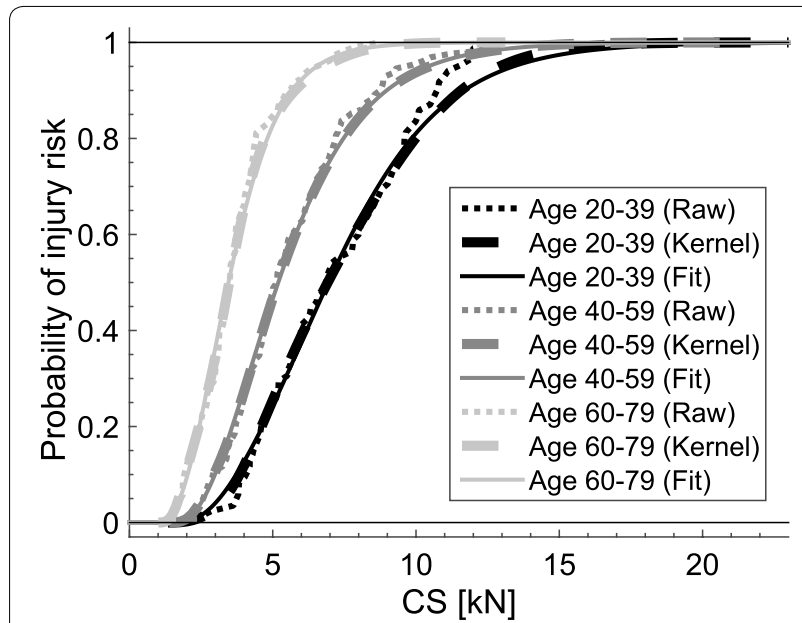

Fig. 4 Risk curves for three generations, fitted to Eq. 8. CS, compressive strength

Table 2 Resultant coefficients of the risk curve for three generations, fitted to Eq. 8

\begin{tabular}{llll}
\hline Age group (years) & $\boldsymbol{a}_{\mathbf{0}}$ & $\boldsymbol{a}_{\mathbf{1}}$ & $\boldsymbol{a}_{\mathbf{2}}$ \\
\hline $20-39$ & 16.77 & 2.544 & -0.4166 \\
$40-59$ & 15.00 & 1.953 & -0.4831 \\
$60-79$ & 6.388 & 2.338 & -0.8312 \\
\hline
\end{tabular}

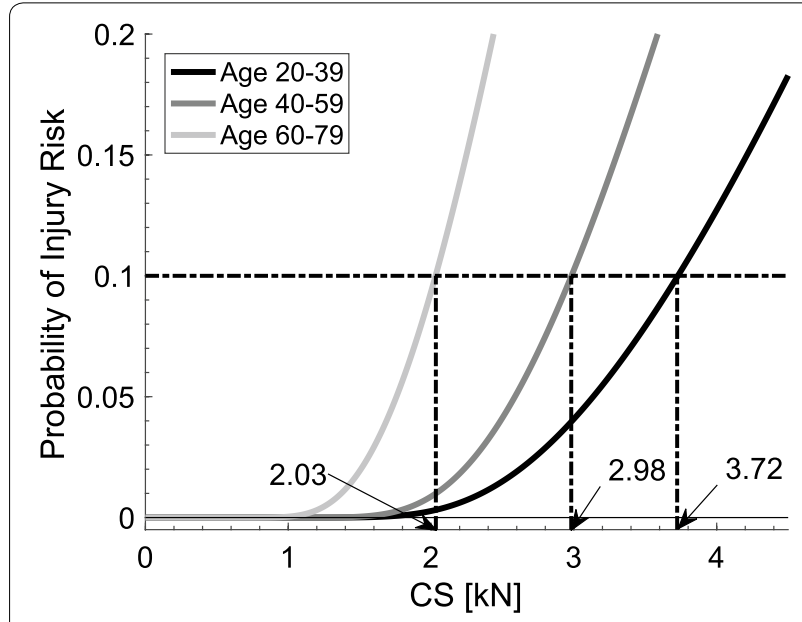

Fig. $510 \%$ risk level of the risk curves for three generations. CS, compressive strength

depending on the age of the user to save on battery power. However, the estimated risk curves of the three generations have distinctive differences; the ages that are at the range limit of two successive ranges may experience large differences in assistive power. Using the risk curve for a specific age may prevent these situations from occurring. Therefore, we derived a risk curve with two variables, namely the two-dimensional risk curve. The variables are $\mathrm{CS}(\mathrm{kN})$ and age (years) in this paper.

The two-dimensional kernel density estimation can be applied to estimate the probability density distribution without grouping the ages:

$$
\hat{f}_{h}(x, y)=\frac{1}{n h_{x} h_{y}} \sum_{i=1}^{n}\left[K\left(\frac{x-X_{i}}{h_{x}}\right) K\left(\frac{y-Y_{i}}{h_{y}}\right)\right] .
$$

Here, $x$ denotes CS $(\mathrm{kN})$, and $y$ denotes age (years). The log-scaled CS yielded a satisfactory estimation for this case. The two-dimensional kernel density estimation with transformed $(x \rightarrow t(x))$ can be written as follows:

$$
\hat{f}_{h}(x, y)=\frac{1}{n h_{x} h_{y}} \sum_{i=1}^{n}\left[K\left(\frac{t(x)-t\left(X_{i}\right)}{h_{x}}\right) K\left(\frac{y-Y_{i}}{h_{y}}\right)\right] t^{\prime}(x),
$$

where $t(x)=\ln (x)$ in this study. For the estimation of risk for a specific age, the result of the integration of the density along the $x$-axis should be equal to 1 . Therefore, we normalized the equation as follows:

$$
\begin{aligned}
\tilde{f}_{h}(x, y) & =\frac{\hat{f}_{h}(x, y)}{\int_{-\infty}^{\infty} \hat{f}_{h}(x, y) d x}=\frac{\hat{f}_{h}(x, y)}{\hat{f}_{h}(y)} \\
& =\frac{\sum_{i=1}^{n}\left[K\left(\frac{t(x)-t\left(X_{i}\right)}{h_{x}}\right) K\left(\frac{y-Y_{i}}{h_{y}}\right)\right]}{\sum_{i=1}^{n} K\left(\frac{y-Y_{i}}{h_{y}}\right)} \frac{t^{\prime}(x)}{h_{x}} .
\end{aligned}
$$

Hence, the estimated two-dimensional risk curve, is expressed as

$$
\hat{r}_{h}(x, y)=\int_{-\infty}^{x} \tilde{f}_{h}(x, y) d x .
$$

Equation 13 was used for the calculation of the bandwidths $h_{x}$ and, $h_{y}$, given in the work of Bowman and Azzalini [33]:

$$
h_{x}=\left(\frac{1}{n}\right)^{1 / 6} \sigma_{x}, \quad h_{y}=\left(\frac{1}{n}\right)^{1 / 6} \sigma_{y} .
$$

For the calculation of $\sigma$, Eq. 7 was used for both the $x$-axis and $y$-axis directions.

Since the risk curve in Eq. 12 requires the input of data shown in Fig. 2, in this form, it is inconvenient from the 


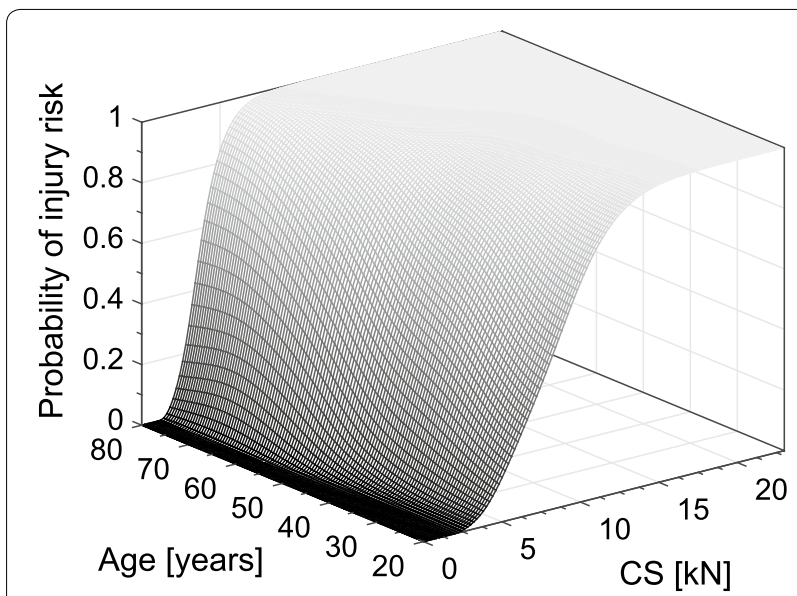

Fig. 6 Risk curve for specific ages estimated via the kernel density estimation. CS, compressive strength

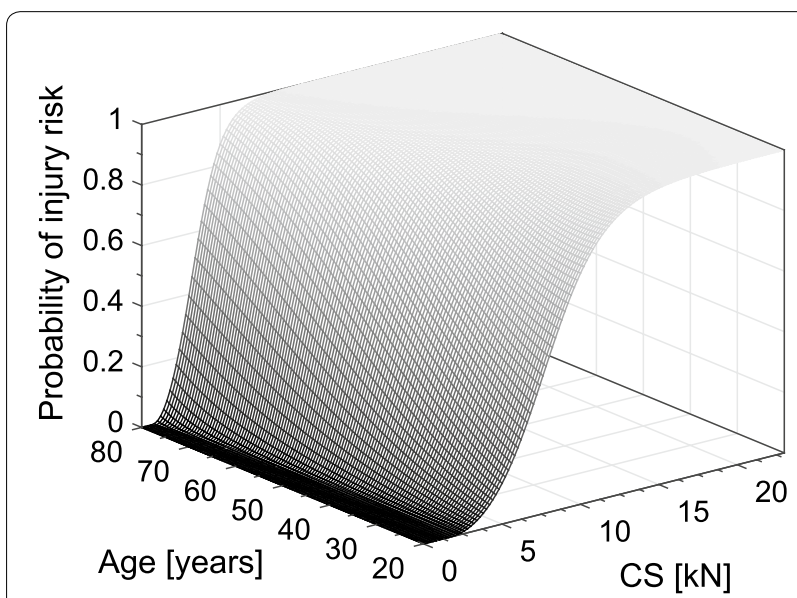

Fig. 7 Risk curve for specific ages, using Eq. 14, with $R^{2}=0.9955$. CS, compressive strength

viewpoint of usability. Therefore, we consider fitting it to the following function:

\section{Analysis of risk curves}

Three types of risk curves were introduced, namely for the general population, for three generations, and for specific ages. Regarding the risk curves for three generations in Fig. 4, certain differences could be observed in the 20-39-year age group curve, between the fitted curve and the raw curve. This may have resulted from a shortage in data. In the lower risk level of our interest, it can be certain that there is no critical problem of safety with the fitted risk curve that resulted in a higher risk than with the raw ones. Additionally, from Fig. 5, the NIOSH criterion of $3.4 \mathrm{kN}$ can be placed between the 20-39-year curve and 40-59-year curve, which agrees with the suggestion by Jäger and Luttmann [21].

Regarding the specific-age risk curve, there were certain regions where lower age presented higher risks than higher age for the same CS value, which does not agree with the generic characteristics of higher age, namely, being at a higher risk. As the region was placed in quite a high risk level, close to 1 , safety should not pose a problem. In Fig. 8, the contour graph of the low risk level is presented. The raw contour was based on raw risk curves for each age, which were derived based on data with an age tolerance of \pm 5 years (11-year width). It can be assumed that the discontinuity presented in the raw contour was caused by the lack of data. Comparing fitted curves with raw curves, the fitted risk curve had the tendency to estimate a lower risk in elderly individuals and a higher risk in young individuals. The lower risk estimation indicates having a problem from a validation viewpoint.

These characteristics can be regarded as the result of the cost functions providing least-squares errors for all of the risk levels. Hence, we introduced a weighted risk curve on the low risk level (Fig. 9, Table 4) for a specific age. The fitting method is described in detail in Appendix B. A contour graph for this risk curve is shown in Fig. 10. By comparing Figs. 8 and 10, the low-risk-level

$$
r(x, y)=\frac{1}{1+\exp \left\{\frac{\left(A_{0}+A_{1} y+A_{2} y^{2}\right)+\left(A_{3}+A_{4} y+A_{5} y^{2}\right) x^{2}+\left(A_{6}+A_{7} y+A_{8} y^{2}\right) x^{3}}{x^{2}}\right\}} .
$$

Fig. 6 shows the estimated risk curve for specific ages via the kernel density estimation,while Fig. 7 shows the risk curve for specific ages fitted to Eq. 14. Table 3 lists the coefficients fitted to the risk curve (Eq. 14). weighted graph reproduced the characteristics of the raw contour more satisfactorily than the non-weighted one in the lower risk level for elderly individuals. Therefore, we can conclude that for the risk curve for

Table 3 Resultant coefficients of the risk curve for specific ages, fitted to Eq. 14

\begin{tabular}{lllllllll}
\hline $\boldsymbol{A}_{\mathbf{0}}$ & $\boldsymbol{A}_{\mathbf{1}}$ & $\boldsymbol{A}_{\mathbf{2}}$ & $\boldsymbol{A}_{\mathbf{3}}$ & $\boldsymbol{A}_{\mathbf{4}}$ & $\boldsymbol{A}_{\mathbf{5}}$ & $\boldsymbol{A}_{\mathbf{6}}$ & $\boldsymbol{A}_{\mathbf{7}}$ & $\boldsymbol{A}_{\mathbf{8}}$ \\
\hline 6.580 & 0.5793 & -0.007871 & 7.486 & -0.2254 & 0.002175 & -0.9376 & 0.02866 & -0.0003792 \\
\hline
\end{tabular}




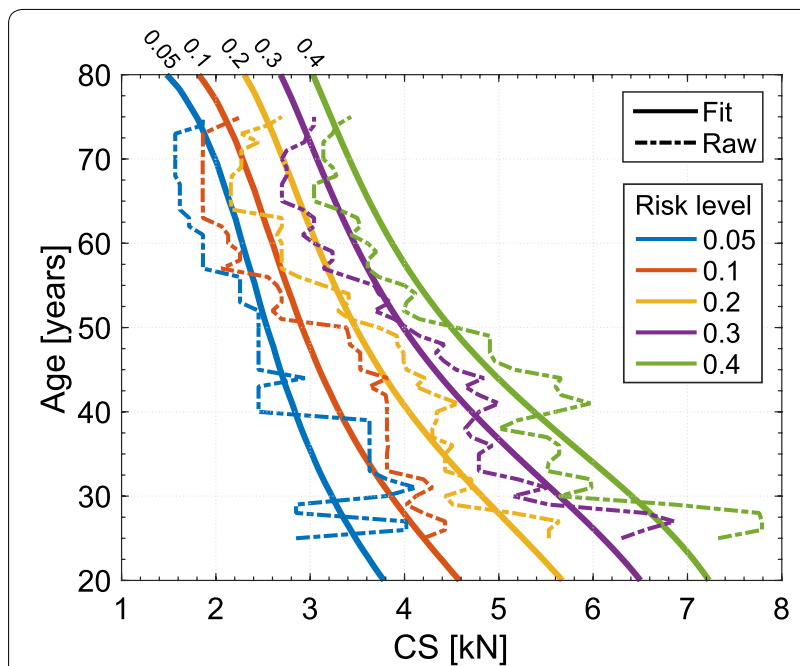

Fig. 8 Contour graph of the risk curves for specific ages. CS, compressive strength

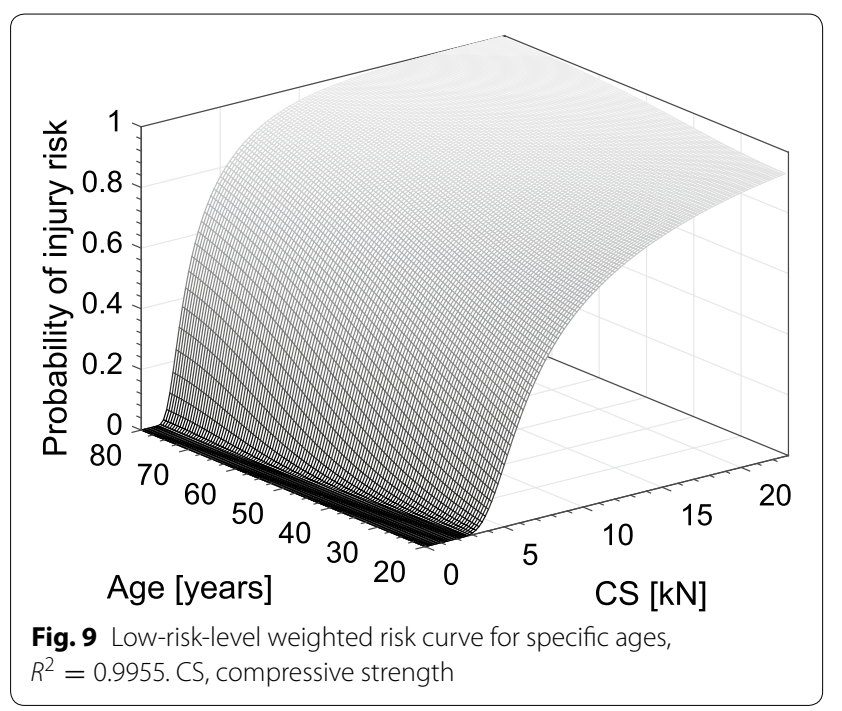

a specific age, the low-risk-level weighted risk curve should be employed for the safety of the elderly. However, it should be noted that even the low-risk-level weighted risk curve estimated a 5-10\% lower risk level for the case of elderly individuals than the raw graph. Additionally, it should be also noted that the 5\% risk level for younger individuals estimated lower risk than that in the raw-data graph.

\section{Discussion}

The injury risk curves introduced in this study could be used for designing and evaluating manual work, by integrating the results of studies that estimated the compressive force on the lumbar spine [34-36]. When the

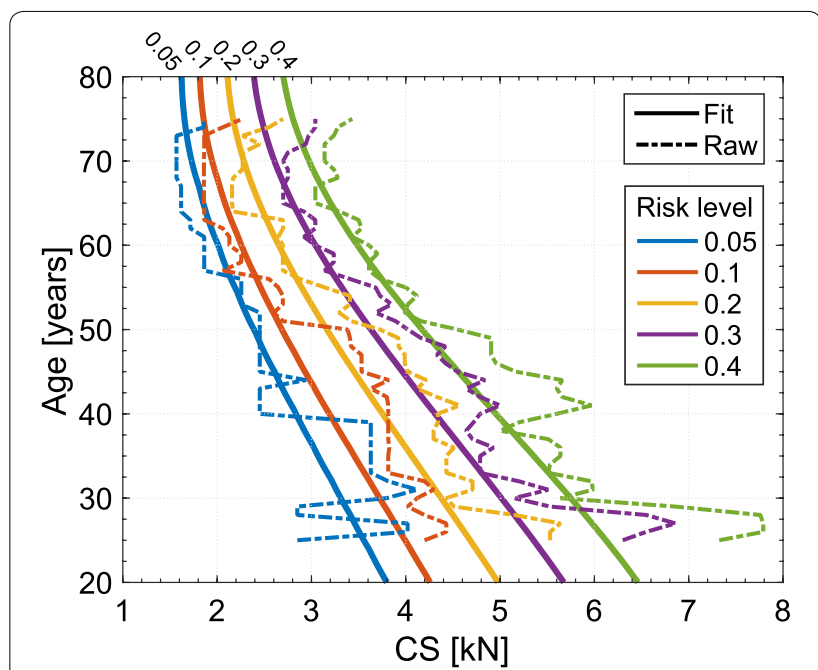

Fig. 10 Contour graph of the low-risk-level weighted risk curves for specific ages. CS, compressive strength

compressive forces on the lumbar spine were estimated, the probability of LBP risk could be easily computed by substituting the values into the $x$ (CS) of Eq. 8 or 14 .

The evaluation of manual work based on lumbar compressive forces can be seen in the works of Merryweather et al. [34], Arjmand et al. [12, 35], Gallagher and Heberger [14], and Ray et al. [13]. Most of these works refer to NIOSH lumbar compressive force of $3.4 \mathrm{kN}$ as a criterion; however, our injury risk curves demonstrated a higher risk for this value for individuals with higher ages. Using the $3.4 \mathrm{kN}$ of compressive force and the low-risklevel weighted injury risk curve for a specific age, such as for a 20-year-old person, the probability of injury risk was estimated to be $2.1 \%$, in contrast to $56 \%$ for a 79 -year-old person. This indicates the need for re-revising the widely used RNLE (the equation for calculating RWL) [6] and including age as one of the equation components.

The CS value according to age may be affected by certain other factors. One of the considered factors was race. The CS values of Japanese people (Asians) can be obtained from the work of Sonoda [37]. The Mann-Whitney U test [38] was performed by Westerners (Fig. 2) versus Asians (Table 5) for each of the three generations (20-39, 40-59, and 60-79 years). No significant difference was found from the test $(p=0.8464, p=0.4385$, and $p=0.1981$, respectively, which were all greater than 0.1). These results imply the effect of racial differences, but not more significant than that of age. It should be also noted that these statistical tests were not entirely unbiased, due to limited data (e.g., insufficient data from Asian individuals, data from Sonoda [37] might be mean values). Further studies should be performed to draw a realistic conclusion. 
Table 4 Resultant coefficients of the low-risk-level weighted risk curve for specific ages, fitted to Eq. 14

\begin{tabular}{lllllllll}
\hline $\boldsymbol{A}_{\mathbf{0}}$ & $\boldsymbol{A}_{\mathbf{1}}$ & $\boldsymbol{A}_{\mathbf{2}}$ & $\boldsymbol{A}_{\mathbf{3}}$ & $\boldsymbol{A}_{\mathbf{4}}$ & $\boldsymbol{A}_{\mathbf{5}}$ & $\boldsymbol{A}_{\mathbf{6}}$ & $\boldsymbol{A}_{\mathbf{7}}$ & $\boldsymbol{A}_{\mathbf{8}}$ \\
\hline 80.16 & -1.775 & 0.01109 & 0.000 & 0.000 & 0.000 & -0.1439 & 0.002355 & $-5.608 \mathrm{e}-05$ \\
\hline
\end{tabular}

Table 5 Compressive strength (kg) for Japanese people [37]

\begin{tabular}{llll}
\hline Ages & $\mathbf{2 0 - 3 9}$ & $\mathbf{4 0 - 5 9}$ & $\mathbf{6 0 - 7 9}$ \\
\hline L1 & 755 & 477 & 291 \\
L2 & 740 & 481 & 389 \\
L3 & 744 & 484 & 310 \\
L4 & 719 & 481 & 339 \\
L5 & 691 & 461 & 311 \\
\hline
\end{tabular}

Data may be the average and not the measured values (not mentioned explicitly in the literature)

Increasing the number of parameters related to CS could enhance the usefulness of the risk curves. In this study, we focused on one parameter, namely age, which significantly influenced the CS values, due to limited data. The gender factor may be the first candidate for a supplemental parameter to improve the risk curves. Jäger and Luttmann [21] suggested that age- and gender-related analyses should be conducted. Furthermore, Ebbesen et al. [29] reported that in the correlation between CS and age, males generally presented with higher CS values than females. Thus, age- and gender-related risk curves should be introduced in future studies, and a higher number of CS measurement studies should be analyzed.

Some researchers used the lumbar spine shear force as a biomechanical criterion in addition to the compressive force $[9,12]$. A limit of $1.0 \mathrm{kN}$ was suggested in some earlier studies [39-41]. In this study, we focused on compressive force since RNLE is currently used as the standard, which is based on compressive force [7]. However, we believe shear force should also be considered as a risk factor. Therefore, injury risk curves for shear force should also be introduced in future studies.

Regarding the development of physical assistant robotic devices, the lumbar compressive force with the assistance of the devices can be estimated using methods such as inverse dynamics [8]. The net amount of risk reduced by the devices can be computed by referring to the risk curve. This curve may help developers in designing the devices. More clearly, the curve may enable them to set a specific target for reducing the risk level and the age of individuals. Additionally, the risk curves could be used directly in the safety standards of physical assistant robots such as ISO or JIS. For example, the Lumbar Compression Reduction (LCR) in the current version of the associated JIS standard for physical assistant robots with lumbar supports [4] can be updated to describe a reduction of the probability of risk taking into account the data of the risk curves we obtained. In parallel, desirable risk levels for physical assistant robots should be elaborately determined to pertain the safety of users. Since the detailed usage of the risk curves must depend on how they are evaluated in their use scenarios, the risk level should also be determined under each of their use scenarios in consideration of the age groups that vary. We may be able to exemplify such an effective use of the risk curves as follows: Some use scenario can accept a $10 \%$ for all age groups, while some other scenario which may conclude that underestimation of the risk curve for elderly cannot be accepted due to the possible use of such devices by elderly, and therefore select $10 \%$ for the youth and $5 \%$ for the elderly.

\section{Conclusions}

In this study, the risk estimation of LBP for manual lifting tasks was performed based on the CS of the lumbar spine. Analyses for low risk levels were possible by introducing nonlinear injury risk curves. For individual differences, an age factor was primarily considered, and risk curves for three generations as well as two-dimensional injury risk curves for specific ages were produced. Regarding the risk curves, a quantitative assessment of the use of physical assistive devices and an evaluation of manual handling tasks are rendered possible when the compressive force on the lumbar spine is estimated. A race factor was also discussed by comparing between Westerners and Asians (Japanese) in three age groups. However, results indicated that the effect of race was not more significant than that of age, although limited data make this finding inconclusive.

\section{Acknowledgements}

Primary support for this work was provided by the Robotic Devices for Nursing Care Project of Ministry of Economy, Trade and Industry (METI) and Japan Agency for Medical Research and Development (AMED). We would like to express our gratitude to Dr. Kazuyuki Iwakiri at the National Institute of Occupational Safety and Health, Japan, Dr. Yasuhiro Akiyama, a research associate, and Dr. Shogo Okamoto, an associate professor at the Nagoya University for their advice regarding this research. Special thanks are also addressed to Mr. Hiroki Ishimoto, an undergraduate student at Nagoya University. 
Authors' contributions

NK carried out the main part of this study and drafted the manuscript. YY contributed concepts of this study and revised the manuscript. DI contributed associated conventional work to introduce. All authors read and approved the final manuscript.

\section{Funding}

This research was supported by the Robotic Devices for Nursing Care Project of Japanese Ministry of Economy, Trade and Industry (METI) and Japan Agency for Medical Research and Development (AMED) under Grant METI-20130524 to AMED-15he1202004h0103.

\section{Competing interests}

The authors declare that they have no competing interests.

$$
\begin{aligned}
& a_{0} \approx A_{0}+A_{1} y+A_{2} y^{2}, \\
& a_{1} \approx A_{3}+A_{4} y+A_{5} y^{2}, \\
& a_{2} \approx A_{6}+A_{7} y+A_{8} y^{2} .
\end{aligned}
$$

Therefore, the functions $r$ and $f$ can be rewritten as follows, using $x$ and $y$ :

$$
r(x, y)=\frac{1}{1+\exp \{f(x, y)\}},
$$

$$
f(x, y)=\frac{\left(A_{0}+A_{1} y+A_{2} y^{2}\right)+\left(A_{3}+A_{4} y+A_{5} y^{2}\right) x^{2}+\left(A_{6}+A_{7} y+A_{8} y^{2}\right) x^{3}}{x^{2}} .
$$

\section{Appendix A: The form of function for the risk curve}

The function for the risk curve requires the following conditions: $\lim _{x \rightarrow+0} r(x)=+0$ and $\lim _{x \rightarrow \infty} r(x)=1$. Logistic functions are known to have similar profiles of satisfying these conditions. In addition to the above conditions, we can design a function as in below, by considering the following conditions: $\lim _{x \rightarrow-0} r(x)=+0$, and $\lim _{x \rightarrow-\infty} r(x)=+0$. The additional conditions were used to decrease the degree of freedom but were not necessary since $x \geq 0$ in this study.

\section{Appendix B: The fitting conditions for the low-risk-level weighted risk curve for specific ages}

The constrained nonlinear optimization technique was used for the fitting. The coefficients of the risk curve $\boldsymbol{A}=\left[A_{0}, \ldots, A_{8}\right]^{T}$ were determined by solving the following optimization problem:

$$
\boldsymbol{A}^{*}=\underset{\boldsymbol{A}}{\arg \min } \sum_{i, j}\left[\left\{\hat{r}_{h}\left(\boldsymbol{A}, x_{i}, y_{j}\right)-r\left(\boldsymbol{A}, x_{i}, y_{j}\right)\right\}^{2}+\delta\left\{1-\left(\frac{\hat{r}_{h}\left(\boldsymbol{A}, x_{i}, y_{j}\right)}{r\left(\boldsymbol{A}, x_{i}, y_{j}\right)}\right)^{2}\right\}^{2}\right]
$$

$$
\begin{aligned}
& r(x)=\frac{1}{1+\exp \{f(x)\}} . \\
& f(x)=\frac{\sum_{i=0}^{m} a_{i} x^{2 i}+\sum_{j=1}^{n} a_{m+j} x^{2 m+2 j-1}}{x^{2 m}} \\
& \begin{cases}a_{k} \geq 0 & (k=0, \ldots m) \\
a_{k} \leq 0 & (k=m+1, \ldots, m+n) .\end{cases}
\end{aligned}
$$

Here, $m=1$ and $n=1$ were chosen in the study:

$$
f(x)=\frac{a_{0}+a_{1} x^{2}+a_{2} x^{3}}{x^{2}} .
$$

For the two-dimensional risk curve, the transition of coefficients $a_{0}, a_{1}$, and $a_{2}$ along $y$-axis was estimated by fitting them with $\hat{r}\left(x, y^{*}\right)$. Here, $y^{*}$ denotes the discretized value of $y \in[20,79]$. The estimated coefficients $a_{0}(y)$, $a_{1}(y)$, and $a_{2}(y)$ were fitted to the lowest polynomial function that can express the transitions, which were quadratic equations in this paper. A least-squares method was used for the fitting: subject to

$$
\begin{aligned}
& a_{0}\left(A, y_{j}\right) \geq 0, \quad a_{1}\left(A, y_{j}\right) \leq 0, \quad a_{2}\left(A, y_{j}\right) \leq 0, \\
& \left.\frac{\partial r(A, x, y)}{\partial y}\right|_{x=x_{i}, y=y_{j}} \geq 0
\end{aligned}
$$

where

$$
\delta= \begin{cases}1 & \left(0.02<\hat{r}_{h}\left(\boldsymbol{A}, x_{i}, y_{j}\right)<0.3\right) \\ 0 & \left(\hat{r}_{h}\left(\boldsymbol{A}, x_{i}, y_{j}\right) \leq 0.02, \quad \hat{r}_{h}\left(\boldsymbol{A}, x_{i}, y_{j}\right) \geq 0.3\right) .\end{cases}
$$

The first term of the cost function, Eq. 22, represents the least-squares error, and the second term represents the weighting in the low risk level. The inequality constraints, Eq. 23, represent the constraints for the risk curve, Eq. 17. The final inequality constraints, Eq. 24, represent the generic tendency of the lower ages, namely that they present a lower risk than the higher ages for the same CS value; $x_{i}$ and $y_{j}$ represent the $i$ th and $j$ th numbers of the discretized $x$ and $y$, respectively. Eq. 25 describes $\delta$, 
which determines the weighting conditions for lower risk fitting. Moreover, $\hat{r}_{h}$ represents the two-dimensional risk curve estimated by the kernel density estimation, Eq. 12; $r$ represents the function of a two-dimensional risk curve, Eq. 14. The fmincon function in the optimization toolbox of MATLAB (MathWorks) was used obtaining for the solution of the optimization problem.

Received: 13 December 2018 Accepted: 3 September 2019

Published online: 18 September 2019

\section{References}

1. Fujimura T, Yasuda N, Ohara H (1995) Work-related factors of low back pain among nursing aides in nursing homes for the elderly. J Occup Health 37(2):89-98

2. Hara H, Sankai Y (2010) Development of HAL for lumbar support. In: SCIS \& ISIS, vol. 2010. Japan Society for Fuzzy Theory and Intelligent Informatics, pp 416-421

3. Imamura Y, Tanaka T, Ayusawa K, Yoshida E (2014) Verification of assistive effect generated by passive power-assist device using humanoid robot. In: System integration (SII), 2014 IEEE/SICE international symposium on, pp 761-766. IEEE

4. Japanese Standards Association (2017) Personal care robots-part 1: physical assistant robots for lumbar support. Standard JIS B 8456-1:2017

5. NIOSH (1981) Work practices guide for manual lifting. Technical Report, pp 81-122

6. Waters TR, Putz-Anderson V, Garg A, Fine LJ (1993) Revised NIOSH equation for the design and evaluation of manual lifting tasks. Ergonomics 36(7):749-776

7. Lu M-L, Putz-Anderson V, Garg A, Davis KG (2016) Evaluation of the impact of the revised national institute for occupational safety and health lifting equation. Hum Factors 58(5):667-682

8. Chaffin D, Andersson G, Martin B (2006) Occupational biomechanics, 4th edn. Wiley-Interscience, New York

9. Ulrey BL, Fathallah FA (2013) Subject-specific, whole-body models of the stooped posture with a personal weight transfer device. J Electromyogr Kinesiol 23(1):206-215

10. Potvin JR (2014) Comparing the revised NIOSH lifting equation to the psychophysical, biomechanical and physiological criteria used in its development. Int J Ind Ergon 44(2):246-252

11. Lu S-Y, Ing Y-S, Lee C-L, Pan Y-T (2014) Development and validation of a posture driven tool to estimate the hazards of manual lifting. Adv Phys Ergon Hum Factors Part I 14:299

12. Arjmand N, Amini M, Shirazi-Adl A, Plamondon A, Parnianpour M (2015) Revised NIOSH lifting equation may generate spine loads exceeding recommended limits. Int J Ind Ergon 47:1-8

13. Ray PK, Parida R, Sarkar S (2015) Ergonomic analysis of construction jobs in india: a biomechanical modelling approach. Proc Manuf 3:4606-4612

14. Gallagher S, Heberger JR (2015) The effects of operator position, pallet orientation, and palletizing condition on low back loads in manual bag palletizing operations. Int J Ind Ergon 47:84-92

15. Afshari D, Kord S, Latifi SM, Mardi G et al (2016) The adequacy of NIOSH equation to to determine the rate of stresses exerted on the back based on compressive force estimation. Jundishapur J Health Sci 9(1):e37726

16. Gallagher S, Mayton A (2007) Back injury control measures for manual lifting and seat design. Min Eng 59(12):41-49

17. Bogduk N (2005) Clinical anatomy of the lumbar spine and sacrum, 4th edn. Churchill Livingstone, London

18. Gallagher S (2008) Reducing low back pain and disability in mining. DHHS (NIOSH) Publication, Pittsburgh
19. Adams MA, Freeman BJ, Morrison HP, Nelson IW Dolan P (2000) Mechanical initiation of intervertebral disc degeneration. Spine 25(13):1625-1636

20. Genaidy A, Waly S, Khalil T, Hidalgo J (1993) Spinal compression tolerance limits for the design of manual material handling operations in the workplace. Ergonomics 36(4):415-434

21. Jäger M, Luttmann A (1999) Critical survey on the biomechanical criterion in the NIOSH method for the design and evaluation of manual lifting tasks. Int J Ind Ergon 23(4):331-337

22. Newman JA, Tylko S, Miller T (1994) Toward a comprehensive biomechanical injury cost model. Accid Anal Prev 26(3):305-314

23. Rupp JD, Flannagan CA, Kuppa SM (2010) An injury risk curve for the hip for use in frontal impact crash testing. J Biomech 43(3):527-531

24. Brinckmann P, Biggemann M, Hilweg D (1988) Fatigue fracture of human lumbar vertebrae. Clin Biomech 3:23

25. Hansson T, Roos B, Nachemson A (1980) The bone mineral content and ultimate compressive strength of lumbar vertebrae. Spine 5(1):46-55

26. Hutton W, Adams M (1982) Can the lumbar spine be crushed in heavy lifting? Spine 7(6):586-590

27. Hansson T, Roos B (1980) The influence of age, height, and weight on the bone mineral content of lumbar vertebrae. Spine 5(6):545-551

28. Adams M, Hutton W (1982) Prolapsed intervertebral disc: a hyperflexion injury. Spine 7(3):184-191

29. Ebbesen EN, Thomsen JS, Beck-Nielsen H, Nepper-Rasmussen HJ, Mosekilde L (1999) Age-and gender-related differences in vertebral bone mass, density, and strength. J Bone Miner Res 14(8):1394-1403

30. Eie N (1966) Load capacity of the low back. J Oslo City Hosp 16(4):73-98

31. Brinckmann P, Frobin W, Hierholzer E, Horst M (1983) Deformation of the vertebral end-plate under axial loading of the spine. Spine 8(8):851-856

32. Parzen $E$ (1962) On estimation of a probability density function and mode. Ann Math Stat 3:1065-1076

33. Bowman AW, Azzalini A (1997) Applied smoothing techniques for data analysis: the kernel approach with S-plus illustrations. Oxford University Press, New York

34. Merryweather AS, Loertscher MC, Bloswick DS (2009) A revised back compressive force estimation model for ergonomic evaluation of lifting tasks. Work 34(3):263-272

35. Arjmand N, Plamondon A, Shirazi-Adl A, Lariviere C, Parnianpour M (2011) Predictive equations to estimate spinal loads in symmetric lifting tasks. J Biomech 44(1):84-91

36. Rajaee MA, Arjmand N, Shirazi-Adl A, Plamondon A, Schmidt H (2015) Comparative evaluation of six quantitative lifting tools to estimate spine loads during static activities. Appl Ergon 48:22-32

37. Sonoda T (1962) Studies on the strength for compression, tension and torsion of the human vertebral column. J Kyoto Pref Med Univ 71:659-702 (in Japanese)

38. Mann HB, Whitney DR (1947) On a test of whether one of two random variables is stochastically larger than the other. Ann Math Stat 18:50-60

39. McGill SM (1997) The biomechanics of low back injury: implications on current practice in industry and the clinic. J Biomech 30(5):465-475

40. McGill S, Norman R, Yingling V, Wells R, Neumann P (1998) Shear happens! suggested guidelines for ergonomists to reduce the risk of low back injury from shear loading. In: Proceedings of the 30th Annual Conference of the Human Factors Association of Canada, pp 157-161

41. Gallagher S, Marras WS (2012) Tolerance of the lumbar spine to shear: a review and recommended exposure limits. Clin Biomech 27(10)·973-978

\section{Publisher's Note}

Springer Nature remains neutral with regard to jurisdictional claims in published maps and institutional affiliations. 